\title{
|||||||||||||||||
}

PB97-184097

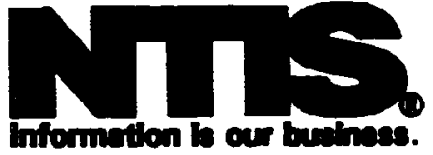

\section{DIFFUSE-INTERFACE METHODS IN FLUID MECHANICS}

(U.S.) SOUTHAMPTON UNIV. (ENGLAND)

MAY 97

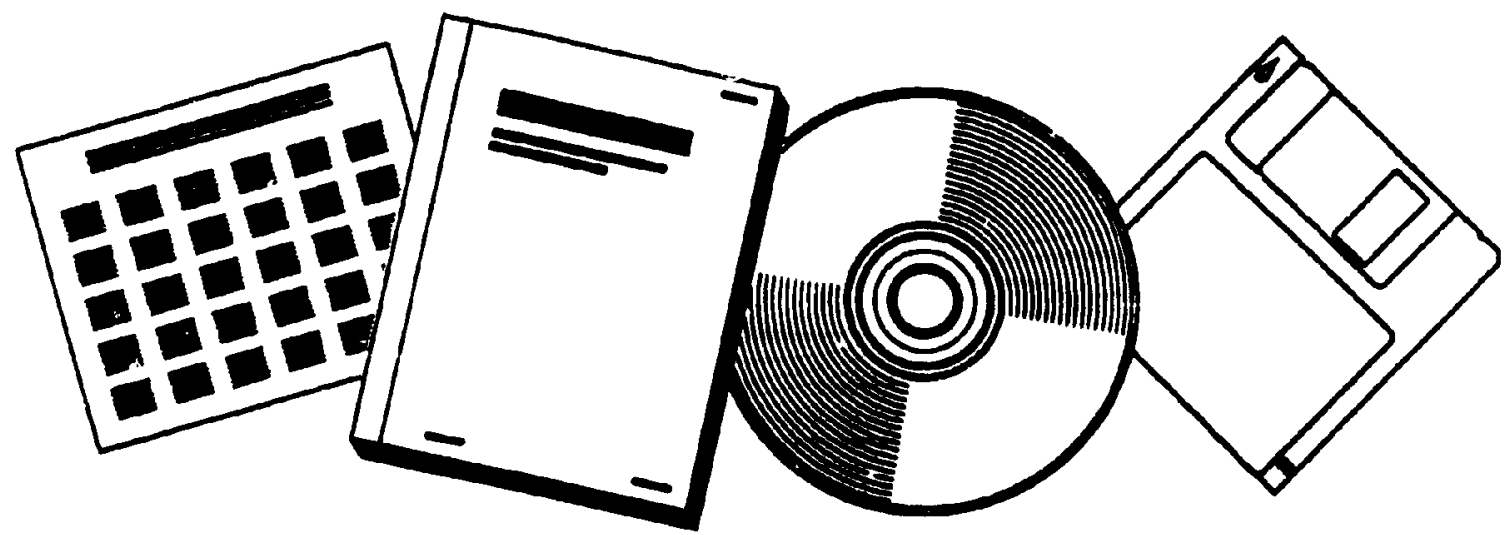

U.S. DEPARTMENT OF COMMERCE

National Technical Information Service 



\section{Diffuse-Interface Methods in Fluid Mechanics}

D. M. Anderson
Q. B. Mefadden

\section{A. A. Wheeler}

Faculty of Mathematical Studies

University of Southampton

Highfield, Southampton SO17 1BJ, UK

U.S. DEPARTMENT OF COMMERCE

Technolugy Administration

National Institute of Standards

and Technology

Gaithersburg, MD 208990001 


\section{MANUSCRIPT REVIEW AND APPROVAL}

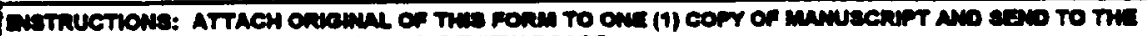

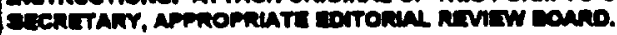

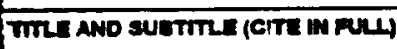

Diffuse-Interface Methods in Fluid Mechanics

\begin{tabular}{|c|c|}
\hline cowtract on andur mumest & 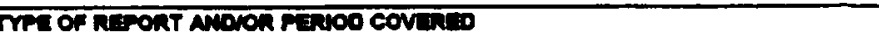 \\
\hline 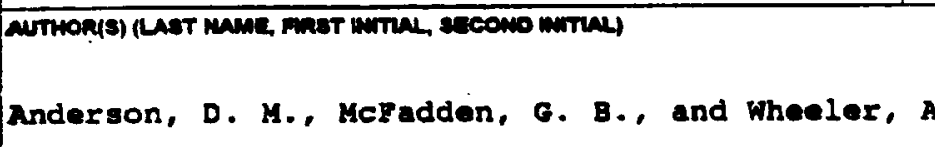 & 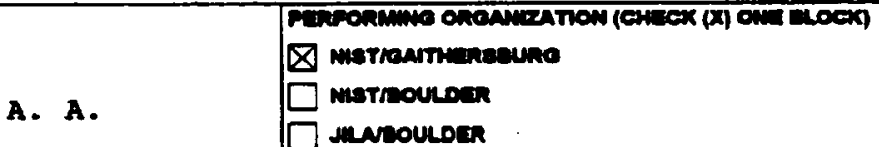 \\
\hline
\end{tabular}

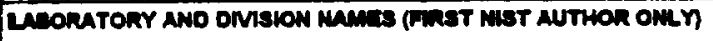

Information Technology Laboratory, Kathematical and Computational Sciences Diviaion

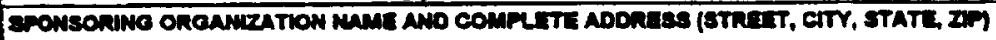

Nist osestory tio. NIST.

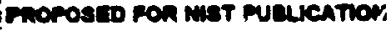

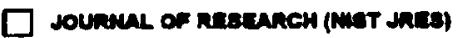 \\ J. mHY. \& CHEM. Rer. DATA (JPCRD) MunOSOOK (MWST HO) SPLCWL MULLCATION (MNOT SP) TECHNICAL MOTE (MLST TN)}

monooramp (most imin)

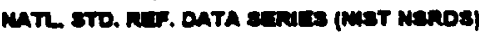
Fidesul inw. Mnocesse. stos. (nust mpa)

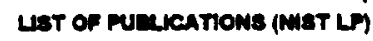

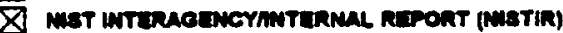

\section{0}

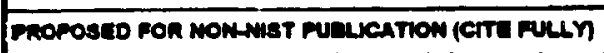

Annual Reviews of Fluid Mechanice

\section{SUAPLIMENTARY MOTES}

mor unvanason

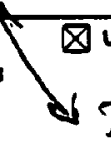

$$
2 \text { flaces of }
$$
$\square$ LeTren cinculan
Eumburo sciance senute moouct stakioande OnNar

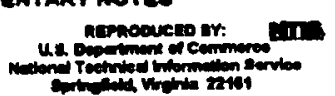

$$
\text { NISTRTSOS }
$$

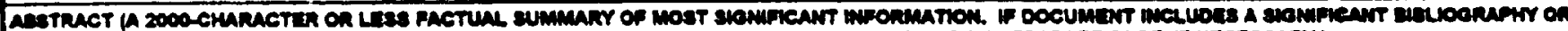

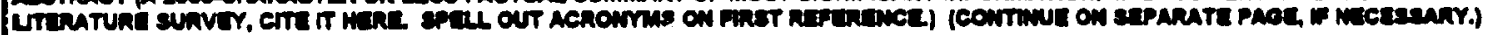

We review the development of diffuee-interface modele of hydrodynamice and their application to a wide variety of interfacial phenomena. These models have been applied auccesefully to situations in which the physical phenomena of interest have associated with them a longth ecale commensurate with the thieknese of the lnterfacial region, (e.g.pnoar-critical

interfacial phenomena or small ecale flowe such as those occurring near contact lines), and fluid flows involving large interface deformatione and/or topologlcal chenges (e.g. breakup and coalescence aseoclated with fluid jets, droplets, and large-deformation weves). We discuse the iseues Involved in formulating diffues-interface modele for single-component and binary fluids. Recent applicatione and computations using these models are discussed in each caee. Further, we addrese levues including sharp-interface analyses that relate these models to the clastical tree-boundary problem, rolated computational approaches to deseribe interfacial phenomena, and related approaches deacribing fuljy-aiscible tlulde.

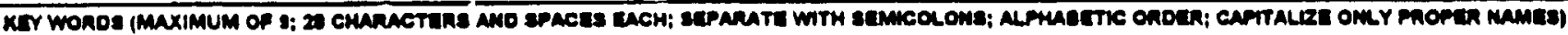

diffuse-interface, phave-fleld; caplilarity, eurface tolloni critical phenomena

\section{AVALAOILITY}

Q UMLIMITEO

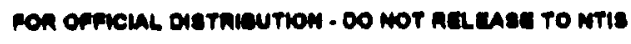

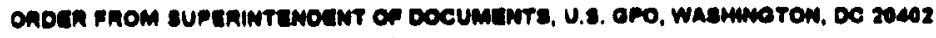
onden nnom nTIs, spanofielo, va z2181
MOTE TO AUTHON(S): W YOU DO WOT MLA THI

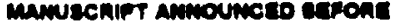
melicatiom. mease check hene. 
NISTIR 6018

\section{Diffuse-Interface Methods in Fluid Mechanics}

\section{M. Anderson \\ Q. B. Mofadden}

\section{A. A. Wheoler}

Faculty of Mathernatical Studies

University of Southampton

Highfield, Southampton SO17 1BJ, UK

U.S. DEPARTMENT OF COMMERCE

Technology Administration

National Institute of Standards

and Technology

Gaithersburg, MO 208990001

May 1997

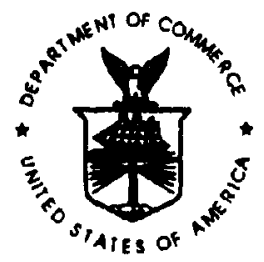

U.S. DEPARTMENT OF COMMERCE William M. Daley, Secretary

TECMNOLOGY ADMINISTAATION

Mary L. Cood, Under Secrotary tor Technolody

NATIONAL INSTITUTE OF STANDARDS

ANO TECHNOLOQY

Robert E. Hebner, Actins Director 


\title{
DIFFUSE-INTERFACE METHODS IN FLUID MECHANICS
}

\author{
D.M. Anderson, G.B. McFadden and A. A. Wheeler* \\ National Institute of Standards and Technology \\ Gaithersburg, MD 20899 \\ USA
}

\begin{abstract}
Absiract
We review the development of diffuse-interface models of hydrodynamics and their application to a wide variety of interfacial phenomena. These models have been applied successfully to situations in which the physical phenomena of interest have associated with them a length scale commensurate with the thickness of the interfacial region, (e.g. near-critical interfacial phenomena or small scale flows such as those occurring near contact lines), and fluid flows involving large interface deformations and/or topological changes (e.g. breakup and coalescence events associated with fluid jets, droplets, and large-deformation waves). We discuss the issues involved in formulating diffuse-interface models for single-component and binary fluids. Recent applications and computations using these models are discussed in each case. Further, we address issues including sharp-interface analyses that relate these models to the classical freeboundary problem, computational approaches to describe interfacial phenomena, and models of fully-miscible fluids.
\end{abstract}

- Permanent address: Faculty of Mathematical Studies, University of Southampton, Bighfield, Southampton SO17 1BJ, UK. 


\section{Contents}

1 INTRODUCTION 1

2 A SINGLE-COMPONENT FLUID 4

Equilibrium ..................... 5

Non-equilit เum ...................... 7

Applications ........................ 10

3 A BINARY FLUID 13

Non-equilibriurn . . . . . . . . . . . . . . . . . 13

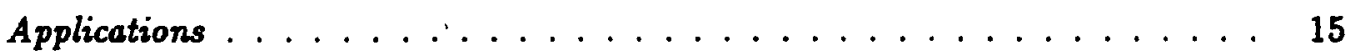

4 RELATED TOPICS 17

Sharp-Interface Limit . . . . . . . . . . . . . . . . . 17

Other Computational Methods . . . . . . . . . . . . . . . . 20

Miscible Fluids . . . . . . . . . . . . . . . . . . 23

5 SUMMARY 25

ACKNOWLEDGEMENTS

IITERATURE CITED 


\section{INTRODUCTION}

The nature of the interface between two fluids has been the subject of extensive investigation for over two centuries. Young, Laplace and Gauss, in the early part of the 1800's, considered the interface between two fluids to be represented as a surface of zero thickness endowed with physical properties such as surface tension. In these investigations, which were based on static or mechanical equilibrium arguments, it was assumed that physical quantities, such as density, vere, in general, discontinuous across the interface. Physical processes such as capillarity occurring at the interface were represented by boundary conditions imposed there (e.g. Young's equation for the equilibrium contact angle or the Young-Laplace equation relating the jump in pressure across an interface to surface tension times curvature). Poisson (1831), Maxwell (1876) and Gibbs (1876) recognized that the interface actually represented a rapid but smooth transition of physical quantities between the bulk fluid values. Gibbs introduced the notion of a dividing surface (a 'surface of discontinuity') and surface excess quantities in order to develop the equilibrium thermodynamics of interfaces. The idea that the interface has a non-zero thickness (i.e. it is diffuse) was further developed in detail by Rayleigh (1892), and van der Waals (1893) who proposed gradient theories for the interface based on thermodynamic principles. In particular, van der Waals gave a theory of the interface based on his equation of state and used it to predict the thickness of the interface which he showed became infinite as the critical temperature is approached. Korteweg (1901) built on these ideas to propose a constitutive law for the capillary stress tensor in terms of the density and its spatial gradients. These original ideas have been developed further and refined over the past century and we turn the reader to the work of Rowlinson (1979) and Rowlinson and Widom (1989), who provide thorough discussions of the historical perspectives and complete references to the early work on interfacial and capillary phenomena.

The notion of a diffuse interface and the use of a capillary stress tensor to model the interface between two fluids and the forces associated with it are of central importance to the topics under consideration in the present paper. Our focus here is on the use of diffuseinterface models which fully couple these notions into a hydrodynamic description. Such 
models have been used to understand physical and hydrodynamic phenomena that occur near a fluid's critical point. Additionally, developments in modern computing technology have stimulated a recent r-surgence in the use of the diffuse-interface models for the computation of flows associated with complex interface morphologies and topological clanges, such as droplet breakup and coalescence and the highly nonlinear development of classical hydrodynamic instabilities.

In the classical fluid mechanical approach, the interface between two immiscible fluids is modeled as a free boundary which evolves in time. The equations of motion which hold in each fluid are supplemented by boundary conditions at the free surface which involve the physical properties of the interface. This formulation results in a free-boundary problem (Lamb 1932, Batchelor 1967, Lighthill 1978, Drazin \& Reid 1981, Davis 1983).

Specifically, in the free-boundary formulation it is assumed that the interface has associated with it a surface tension, which on applying a stress balance at the interface gives rise to the interfacial boundary condition

$$
\left.\sigma \cdot \hat{n}\right|_{-} ^{+}=\gamma \mathcal{K} \hat{n}
$$

which relates the jump in the stress across the interface to the interfacial curvature. Here $\sigma$ is the stress tensor, $\hat{n}$ is the unit vector normal to the interface, $\gamma$ is the surface tension (here assumed to be constant) and $\mathcal{K}$ is the appropriately signed curvature. In addition, an interface between two immiscible fluids is impermeable in which case conservation of mass across the interface gives that

$$
\left.\vec{v} \cdot \hat{n}\right|_{-}=\left.\vec{v} \cdot \hat{n}\right|_{+}=V_{n}
$$

wherc $\vec{v}$ represents the velocity of the fluid and $V_{n}$ is the normal velocity of the interface. Finally, for viscous fluids, there is continuity of tangential velocity across the interface

$$
\left.[\vec{v}-(\vec{v} \cdot \hat{n}) \hat{n}]\right|_{-} ^{+}=0
$$

The free-boundary description has been a very successful model in a wide range of situations, however there are also important instances where it breaks down. In short, as a 
physical model it breaks down when the interfacial thickness is comparable to the length scale of the phenomena being examined. For example, (i) in a near-critical fluid the thickness of the interface diverges at the critical point (Stanley 1971) and consequently the representation of the interface as a boundary of zero thickness may no longer be appropriate, (ii) the motion of a contact line along a solid surface involves a detailed consideration of the fluid motion in the vicinity of the contact line, and may require the treatment of length scales comparable to that of the interface thickness, and (iii) the free-boundary description may not be adequate for situations involving changes in the topology of the interface (e.g. the breakup of a liquid droplet), since these processes fundamentally involve physical mechanisms acting on length scales comparable to the interface thickness. In adjition to the above situations, another difficulty associated with the free-boundary formulation arises in its use in computational settings when the free boundary shape becomes complicated or self-intersecting.

Diffuse-interface models provide an alternative description in the face of these difficulties. Quantities which in the free-boundary formulation are localized to the interfacial surface are recognized to be distributed throughout the interfacial region. For example, surface tension in the classical model is a representation of a distributed stress within the interfacial region. In this spirit a continuum theory of the interface may be developed where the reversible part of the stress tensor, $C$, that is associated with surface tension is expressed in its simplest form as

$$
C \propto\left(\rho \nabla^{2} \rho+\frac{1}{2}|\nabla \rho|^{2}\right) I-\nabla \rho \otimes \nabla \rho
$$

where $\rho$ is the fluid density and the components of the outer product $\nabla \rho \otimes \nabla \rho$ are given by $\left(\partial \rho / \partial x_{i}\right)\left(\partial \rho / \partial x_{j}\right)$. The existence of such a stress tensor, often called the capillary tensor, was first described by Korteweg (1901). The derivatives of the density that appear in the stress tensor arise from the non-local interaction of the molecules within the interface. In this situation, the density $\rho$ is the variable which distinguishes the bulk fluids and the intervening interface. In this role, it is known as the order parameter. In contrast, for a binary fluid undergoing spinodal decomposition, the composition $c$ naturally plays the role of an order parameter (Cahn \& Hilliard 1958). Alternatively it is possible that neither the 
density or the composition may be an appropriate or convenient order parameter; such is the case in solidification models (e.g. Caginalp 1985, 1986, Langer 1986). Here it is possible to introduce an alternative order parameter, the so-called phase field $\phi$, to characterize the phases. The phase-field assumes distinct constant values in each bulk phase and undergoes rapid but smooth variation in the interfacial region. The phase field can be regarded as a mathematical device that allows a reformulation of the free-boundary problem and has been used successfully in many instances. In particular, phase-field models of solidification have been used to compute complicated realistic interfacia' structures such as those pres $32 t$ during dendritic growth (Kobayashi 1993, Wheeler et al 1993, Warren \& Boettinger 1995) and Ostwald ripening (Warren \& Murray 1996).

Inherent in diffuse-interface models is an interfacial width which is characterized by the length scale over which the order parameter changes. By considering the asymptotic limit in which the interfacial width is small compared to the macroscopic length scale associated with the motion of the two bulk fluids (i.e. the sharp-interface limit) the diffuse-interface model can be related to the free-boundary problem.

In Section 2 we formulate the diffuse-interface theory of a single-component fluid near its critical point. Here we discuss developments for equilibrium and nonequilibrium situations and also review the applications addressed with this model. In Section 3 we review the developments of these ideas for a binary fluid. In Section 4 we discuss related topics including the sharp-interface limit analyses, computational methods for fluid interfaces and models of miscible fluids.

\section{A SINGLE-COMPONENT FLUID}

Diffuse-interface models of a single-component fluid have been developed largely from the perspective of critical phenomena. While they have been used to study phenomena associated with the critical point, they have also been applied to situations away from the critical point. With this in mind, we shall review in this section the ideas and applications of diffuse-interface models of a single-component fluid. 


\section{Equilibrium}

We begin by first considering the equilibrium state of a non-uniform single-component fluid. We assume that an isothermal fluid near its critical point has associated with it the Helmholtz free energy functional given by

$$
\mathcal{F}=\int_{V}\left[\rho f(\rho, T)+\frac{1}{2} K|\nabla \rho|^{2}\right] d V
$$

where $V$ is a control volume, $f(\rho, T)$ is the bulk free energy density (per unit mass), $K$ is a gradient energy coefficient (assumed for simplicity to be constant) and $T$ is the temperature. In a simple model the term $\rho f \rho, T)$ is assumed to take the form of a double-well with respect to the density below the critical temperature and a single-well above the critical temperature. The square-gradient term is associated with variations of the density and contributes to the free energy excess of the interfacial region, which defines the surface energy (Cahn \& Hilliard 1958). The form of the energy density can be interpreted in the context of statistical mechanics in which the square gradient term arises from attractive long-ranged interactions between the molecules of the fluid and in which the gradient energy coefficient, $K$, can be related to the pair correlation function, (e.g. see Irving \& Kirkwood 1950, Bearman \& Kirkwood 1958, Yang et al 1976, Bongiorno et al 1976, Abraham 1979, de Sobrino \& Peternelj 1982 and Davis \& Scriven 1982 for further details).

The equilibrium conditions are obtained by minimizing $\mathcal{F}$ subject to a constraint of constant mass, $\mathcal{M}$, where

$$
\mathcal{M}=\int_{V} \rho d V
$$

This leads to the Euler-Lagrange equation

$$
K \nabla^{2} \rho-(\rho f)_{\rho}+\lambda=0
$$

where $\lambda$ is the Lagrange multiplier associated with conservation of mass. We observe that the integrand of $\mathcal{F}$ as well as that of the mass constraint are independent of the opatial coordinates. Consequently, it follows from Noether's Theorem (Goldstein 1980) that there 
is a corresponding conservation law given by

$$
\nabla \cdot \boldsymbol{T}=0
$$

where $\boldsymbol{T}$ is a second-rank tensor given by

$$
\boldsymbol{T}=\mathcal{L} \boldsymbol{I}-\nabla \rho \otimes \frac{\partial \mathcal{L}}{\partial(\nabla \rho)},
$$

and $\mathcal{L}=\rho f(\rho, T)+\frac{1}{2} K|\nabla \rho|^{2}-\lambda \rho$. Using the Euler-Lagrange equation (7) to eliminate the Lagrange multiplier we find that

$$
\boldsymbol{T}=\left[-p+K \rho \nabla^{2} \rho+\frac{1}{2} K|\nabla \rho|^{2}\right] \boldsymbol{I}-K \nabla \rho \otimes \nabla \rho,
$$

where $p=\rho^{2} f_{\rho}$ has been identified as the thermodynamic pressure, see, e.g., Callen (1985). Using the divergence theorem, $c$ fuation ( 8 ) may be expressed as

$$
\int_{S} \boldsymbol{T} \cdot \hat{n} d A=0
$$

where $S$ is the boundary of a control volume with unit normal vector $\hat{n}$. This is the generalization to three dimensions of the first integral of the Euler-Lagrange equation (7) in one dimension. Equations (8) and (11) suggest that $\boldsymbol{T}$ represents a stress tensor (up to an additive divergence-free contribution). We show in the next section that $\boldsymbol{T}$ represents the reversible part of the stress tensor. Similar equilibrium conditions have been obtained by Blinowski (1973a, 1973b) from the point of view of elastic fluids. A review article covering a variety of aspects of this and related theories can be found in Davis \& Scriven (1982). It was noted by Dunn \& Serrin (1985) that consistency with nonequilibrium thermodynamics requires a more apecific form for the capillary tensor than that used originally by Korteweg (1901) and later in the mechanical equilibrium theories of Aifantis \& Serrin (1983a, 1983b).

The equilibrium density profile $\rho(z)$ obtained using a van der Waals equation of state (Callen 1985, Rowlinson and Widom 1989) represents a smooth transition from one bulk density to the other (in the two phase region) over a length scale associated with the gradient energy coefficient. Such an interface has associated with it a surface energy given by

$$
\gamma=K \int_{-\infty}^{\infty}\left(\frac{d \rho}{d z}\right)^{2} d z
$$


Non-equilibrium

We now pursue the non-equilibriım situation by outlining a thermodynamic procedure involving local balances of mass, linear momentum, energy and entropy consistent with the inclusion of a square-gradient energy term in the internal energy functional. The total mass, $\mathcal{M}$, total momentum, $\overrightarrow{\mathcal{P}}$, total internal energy, $\mathcal{E}$, and total entropy, $\mathcal{S}$ associated with a material volume $\Omega(t)$ are

$$
\begin{aligned}
\mathcal{M} & =\int_{\Omega(t)} \rho d V \\
\overrightarrow{\mathcal{P}} & =\int_{\Omega(t)} \rho \vec{v} d V \\
\mathcal{E} & =\int_{\Omega(t)}\left(\frac{1}{2} \rho|\vec{v}|^{2}+\rho e(s, \rho)+\frac{1}{2} K_{E}|\nabla \rho|^{2}\right) d V \\
\mathcal{S} & =\int_{\Omega(t)} \rho s d V
\end{aligned}
$$

where $\vec{v}$ is the fluid velocity, $e$ and $s$ are the internal energy and entropy per unit mass, and $K_{E}$ is the gradient (internal) energy coefficient which we assume to be constant. Here we have for simplicity neglected body forces such as gravity. The associated physical balance laws can be expressed as

$$
\begin{aligned}
\frac{d \mathcal{M}}{d t} & =0 \\
\frac{d \vec{p}}{d t} & =\int_{\delta \Omega(t)} m \cdot \hat{n} d A, \\
\frac{d \mathcal{E}}{d t} & =\int_{\delta \Omega(t)}\left[\vec{v} \cdot m \cdot \hat{n}-\vec{q}_{E} \cdot \hat{n}\right] d A, \\
\frac{d S}{d t} & +\int_{\delta n(t)} \vec{q}_{S} \cdot \hat{n} d A=\int_{n(t)} j^{\text {prod } d V \geq 0}
\end{aligned}
$$

where $\delta \Omega(t)$ is boundary of $\Omega(t), \boldsymbol{m}$ is the stress tensor, $\vec{q}_{E}$ and $\vec{q}_{S}$ are the internal energy and entropy fluxes, respectively, and jprod is the volumetric entropy production. The quantities $\boldsymbol{m}, \vec{q}_{\boldsymbol{E}}$ and $\vec{q}_{s}$, which in general include both classical and nonclassical contributions, shall

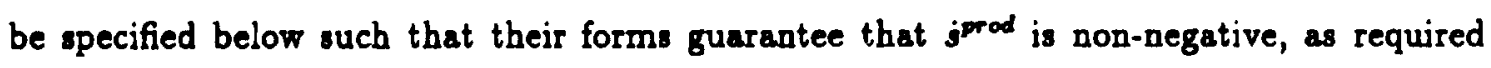
by the Second Law of Thermodynamics. Equation (14a) aimply represents conservation of mass. Equation (14b) states that the change in total momentum is related to the forces 
on the boundary (again note that we have neglected body forces). Equation (14c) states that the change in total energy is related to the rate of working done by the forces on the boundary and also the energy flux through the boundary. Equation (14d) states that the change in total entropy plus the entropy flux through the boundary must be equal to the entropy production. The definitions (13) and balance laws (14) may be manipulated to show that

$$
\dot{s}^{\text {prod }}=\frac{(\boldsymbol{m}-\boldsymbol{T}): \nabla \vec{v}}{T}+\left(\vec{q}_{E}+K_{E} \frac{D \rho}{D t} \nabla \rho\right) \cdot \nabla\left(\frac{1}{T}\right)+\nabla \cdot\left(\vec{q}_{S}-\frac{\vec{q}_{E}}{T}-\frac{K_{E}}{T} \frac{D \rho}{D t} \nabla \rho\right),
$$

where $\boldsymbol{T}$ is the reversible part of the stress tensor given by equation (10) with $K$ replaced by $K_{E}$, and we have used the thermodynamic relationship $d e=T d s+\left(p / \rho^{2}\right) d \rho$. The following specifications ensure that the entropy production is positive

$$
\begin{aligned}
& m=T+T \\
& \vec{q}_{E}=-k \nabla T-K_{E} \frac{D \rho}{D t} \nabla \rho \\
& \vec{q}_{S}=-\frac{k \nabla T}{T}
\end{aligned}
$$

where $k$ is the thermal conductivity and $\tau$ is the viscous stress tensor given in the standard manner as $\tau=\eta(\nabla \cdot \vec{v})+\mu\left(\nabla \vec{v}+\nabla \vec{v}^{2}\right)$, where $\eta$ and $\mu$ are coeffeients of viscosity (e.g. Batchelor 1967). This prescription for $\boldsymbol{m}$ is similar to that postulated by Korteweg (1901). In this formulation it is assumed that the viscosity and thermal conductivity are, in general, functions of the density. We observe that the energy flux $\vec{q}_{E}$ involves both the classical contribution corresponding to the Fourier Law for heat conduction (Carslaw \& Jaeger 1959, Kittel \& Kroemer 1980) and a nonclassical contribution. This nonclassical contribution was referred to as 'interstitial working' by Dunn \& Serrin (1985), who also noted that there is no corresponding nonclassical term in the entropy flux. When a square-gradient term is included in the definition for total entropy (13d), a nonclassical entropy flux arises (e.g. Wang et al 1993, Wheeler et al 1996, Anderson \& McFadden 1996). Using the above forms (16) for the stress tensor and fluxes the local balance laws may then be written as

$$
\frac{D \rho}{D t}=-\rho \nabla \cdot \vec{v}
$$




$$
\begin{aligned}
\rho \frac{D \vec{v}}{D t} & =\nabla \cdot \boldsymbol{m} \\
\rho \frac{D e}{D t} & =\nabla \cdot(k \nabla T)+(-p I+\tau): \nabla \vec{v} \\
\rho T \frac{D s}{D t} & =\nabla \cdot(k \nabla T)+\tau: \nabla \vec{v}
\end{aligned}
$$

These equations describe viscous, compressible, nonisothermal flow. In order to solve these it is necessary to supply an equation of state. In the isothermal situation, for example, one might specify the pressure $p$ through a van der Waals equation of state.

This and similar models have been developed and studied by a number of authors from a variety of perspectives. Fixman (1967) developed a diffuse-interface hydrodynamic model in which the reversible part of the stress tensor was identified using a mechanical principle. In the model of Felderhof (1970), a more general form of the stress tensor was obtained which was compatible with the underlying Lagrangian. Langer \& Turski (1973) derived a diffuse-interface model that they showed, through a 'coarse-graining' argument, could be related to a molecular model. The model employed by Jasnow \& Viñals (1996) was derived using a Hamiltonian description. Jacqmin (1996) described a model which also included a wall potential to model the interaction between the fluid and a solid boundary. Truskinovsky (1993) derived a similar model which also included an additional non-conserved order parameter and its gradients. Antanovskii (1996) presented a derivation of the model based on a maximum entropy principle. The derivation outlined above is most similar to that described by de Sobrino (1976), Dunn \& Serrin (1985), Dunn (1986) and Anderson \& McFadden (1996, 1997). The work of de Sobrino begins in a more general framework and invokes symmetry and invariance principles to simplify the gradient dependence of the stress tensor to that which is described above. Dunn \& Serrin's approach is similar, but mathematically more rigorous and is given from the viewpoint of rational mechanics.

A detailed numerical analysis of a simplified version of the diffuse-interface model has been performed by Affouf and Caflisch (1991), who present numerical solutions representing phase transitions, shocks, and rarefaction waves connecting far-field states and analyze their stability. 


\section{Applications}

CRITICAL POINT SCALING LAWS Extensive analysis using renormalization-group techniques have been performed on a diffuse-interface model, comnonly known in the literature as 'Model H' (Hohenberg \& Halperin 1977), which describes the dynamics of a binary fluid phase transition as well as a single-component fluid near its critical point (e.g. Halperin et al 1974, Siggia et al 1976, and Hohenberg \& Halperin 1977). Such analyses have identified diver: -nt transport coefficients and scaling relations associated with near-critical fluids.

SHEAR FLOWS IN NEAR-CRITICAL FLUIDS Onuki \& Kawasaki (1979) and Onuki et al (1981) have studied the dynamics of a near-critical fluid in a shear flow using a model developed by Kawasaki (1970). They investigated the regime in which the equilihrium correlation length (i.e. the interfacial thickness) exceeds the length scale associated with the shear flow. Among their findings is that the critical fluctuations of classical fluids can be drastically altered (e.g. they can become highly anisotropic) by shear flows. These ideas have also been applied to polymers under shear flows (Helfand \& Fredrickson 1989, Onuki 1989).

CAPILLARY WAVES The diffuse-interface model has been used to study capillary waves (Felderhof 1970, Turski \& Langer 1980, de Sobrino \& Peternelj 1985 and de Sobrino 1985). These authors begin with a diffuse planar interface in equilibrium. They discuss cspilin $y$ waves by means of a linearized theory about the equilibrium state. The linearized governing equations are examined in the long wavelength limit and the dispersion relation for capillary waves obtained from the classical free-boundary problem (e.g. Landau \& Lifshitz 1959) is recovered. These auth'rs recognized the importance of an isentropic treatment to the existence of capillary weves (in the context of a diffuse-interface model) although Felderhof derived the result in the isothermal case as well under somewhat more restrictive conditions.

MOVING CONTACT LINES Seppecher (1996) established the goveraing equations for an isothermal viscous flow near a moving contact line cn a planar solid wall using a diffuse-interface model. In particular, a uniform distribution of 'double forces' was used to 
describe the interaction between the fluid and the wall. In this analysis, the contact-line problem was separated into three regions: an external (outer) region far from the contact line where the classical theory of capillarity applies; an inner region very near the contact line whose dimensions are so small that the thickness of the interface cannot be neglected; and an intermediate region which matches the inner and outer regions. The flow in the inner region is compressible while the flows in the intermediate and outer regions are incompressible. Examples of the density field and flow field in the inner region are shown in Figs. 1 and 2. A key result of this paper was that the force singularity present in classical continuum models of moving contact lines (e.g. Huh \& Scriven 1971, Dussan V. \& Davis 1974, Dussan V. 1979) is not present when the interface is modeled as diffuse. This is attributed to mass transfer across the interfacial region. Additionally, numerical computations reveal a roughly linear increase in the apparent contact angle with the contact-line velocity (no contact-line hysteresis was considered), which agrees with the general trends observed experimentally (see Dussan V. 1979). The moving contact-line problem has also recently been treated computationally by Jacqmin (1996) and is described in the next section.

INTERNAL WAVES IN A NEAR CRITICAL FLUID Anderson \& McI adden (1997) have recently employed the diffuse-interface model to describe internal waves in a nearcritical fluid. These internal waves are present in small (centimeter-scaie) containers owing to the large compressibility of the fluid near the critical point and have been observed experimentally in near-critical Xenon by Berg et al (1996). In the experimental work of Berg et al, internal gravity wave frequencies were measured both above the critical temperature where a single phase exists and below the critical temperature where two phases, separated by an interface, exist. The theoretical development of Berg et al consisted of two separate models; one above the critical temperature and another below the critical temperature. In contrast to these classical hydrodynamic models, the diffuse-interface approach employed by Anderson \& McFadden allows for a single model to be applied both above and below the critical temperature. In their diffuse-interface model, they use a van der Waals equation of state to obtain a base-state density profile. Upon this base-state they introduce linear 
perturbations to calculate internal wave frequencies for temperatures both above and below the critical temperature. Predictions of the internal wave frequency from the diffuse-interface model are compared favorably with experimental data and theoretical results of Berg et al.

DROPLETS AND NUCLEATION Several ? thors have investigated the nucleation of droplets (Blinowski 1974 and Dell'Isola et al 1995, 1996). The focus of this work was to ascertain the effects of interiacial thickness on the $n$ eation conditions of a droplet since, at nucleation, the droplet radius may be comparable to the interfacial thickness. Under these circumstances, the classical Laplace-Gibbs theory for the equilibrium radius of a droplet is called into question. Dell'Isola et al used an equilibrium formulation of a diffuse-interface model developed in earlier work (Dell'Isola \& Kosiński 1993) to study nucleation of spherical droplets. In particular, they noted that for microscopic droplets the difference in mechanical pressures inside and outside the droplet (which is what is measured experimentally) is not the same as the difference between the thermodynamic pressures inside and outside the droplet. The mechanical pressure involves a (stress) contribution from the spatial density variation (e.g. the term in (10) involving $\rho \nabla^{2} \rho$ ) which at the center of the microscopic bubble is important. Based on these ideas, they carefully define quantities such as surface tension and droplet radius in a way which generalize the classical notions. Relationships between surface tension, droplet radius and the critical nucleation radius are obtained using a number of different equations of state. Results for the minimal nucleation radius are compared with experimental measurements.

INSTABILITIES OF PLANAR JETS Nadiga \& Zaleski (1996) studied in the instability of a planar jet of viscous, compressible, isothermal liquid issuing into its surrounding gas phase. They use a van der Waals equation of state to characterize the system. Their calculations focus on the high Reynolds number regime $(R e=800)$ and they investigate the effect of surface tension on the stabilization of the jet.

SPINODAL DECOMPOSITION IN A PURE FLUID Nadiga \& Zaleski (1996) also considered the spinodal decomposition of a single-component fluid rapidly quenched from a temperature above its critical point to a temperature below the critical point. These compu- 
tations are isothermal and two-dimensional. They find that the inclusion of hydrodynamic effects in the model leads to a predicted growth rate which is slightly enhanced from the case when the growth is limited by diffusion. Numerical computations show the domain growth and plots of domain size versus time are presented.

\section{A BINARY FLUID}

\section{Non-equilibrium}

We now consider the situation of a binary fluid which consists of two components $A$ and $B$. We denote the composition of component $A$, expressed as a mass fraction, by $c$. In this setting the composition plays the role of an order parameter which distinguishes the different phases of the fluid and in this way is analogous to the density in the single-component fluid models. As we shall see shortly, however, there are a number of subtle differences between the single-component and binary fluid models.

The governing equations for the flow of a nonisothermal, compressible binary fluid may be developed in a similar manner to those for the single-component case discussed above but involve square-gradient contributions in the internal energy functional from the composition rather than from the density. This procedure gives that the entropy production is

$$
\begin{aligned}
\text { jprod }^{\text {pro }}= & \frac{\left(\boldsymbol{m}-\boldsymbol{T}_{C}\right): \nabla \vec{v}}{T}+\left(\vec{q}_{E}+K_{E} \frac{D c}{D t} \nabla c\right) \cdot \nabla\left(\frac{1}{T}\right)-\vec{q}_{C} \cdot \nabla\left(\frac{\mu_{c}}{T}\right) \\
& +\nabla \cdot\left(\vec{q}_{S}-\frac{\vec{q}_{E}}{T}-\frac{K_{E}}{T} \frac{D c}{D t} \nabla c+\mu_{c} \vec{q}_{C}\right),
\end{aligned}
$$

where $\boldsymbol{T}_{C}$ is the reversible part of the stress tensor given by

$$
\boldsymbol{T}_{C}=\left(-p+\frac{1}{2} K_{E}|\nabla c|^{2}\right) \boldsymbol{I}-K_{E} \nabla c \otimes \nabla c
$$

$\vec{q}_{C}$ is the mass flux of component $A$ and $\mu_{c}$ is the generalized chemical potential given by

$$
\mu_{c}=\frac{\partial e}{\partial c}-\frac{K_{E}}{\rho} \nabla^{2} c
$$

The specification of $\boldsymbol{m}, \vec{q}_{E}, \vec{q}_{S}$ and $\vec{q} C$ to ensure positive entropy production follows in an analogous way to the single-component case. In particular, $\vec{q}_{C}=-D \nabla\left(\mu_{c} / T\right)$, where 
$D$ is positive and represents the diffusion coefficient. Consequently, the equation for the composition is given by

$$
\rho \frac{D c}{D t}=\nabla \cdot\left\{D \nabla\left[\frac{1}{T}\left(\frac{\partial e}{\partial c}-\frac{K_{E}}{\rho} \nabla^{2} c\right)\right]\right\} .
$$

This is well-known Cahn-Hilliard equation, used to model spinodal decomposition by Cahn (1961) and Hilliard (1970), modified to account for fluid motion. The equations governing density, velocity and temperature (energy) are similar to those for a single-component fluid given above by equations (17). Two subtle differences between the binary fluid equations and the single-component fluid equations are worth mentioning. First, the reversible part of the stress tensor $\boldsymbol{T}_{\boldsymbol{C}}$ does not contain the counterpart of the Laplacian term appearing in $\boldsymbol{T}$, see equation (10). This is because the order parameter $c$ is given per unit mass in contrast to the density $\rho$. Second, the order parameter $c$ is governed by the modified Cahn-Hilliard equation (21), whereas the density $\rho$ is governed by the continuity equation (17a).

Details of the derivation of the governing equations for a binary fluid have been discussed by a number of authors. Blinowski (1975) considered the binary fluid case and also more general multi-component systems. Starovoitov (1994) derived a biz 2iy fluid diffuseinterface model using a virtual power method. Antanovskii (1995) derived the equations for nonisothermal, viscous, quasi-compressible flow. His derivation was based on a maximum entropy principle, similar to the approach described here, but used virtual work arguments to identify the forms of the stress tensor and fluxes. Gurtin et al (1996) derived a model for an isothermal, incompressible flow using microforce balance laws. Jasnow \& Viñals (1996) illustrated the derivation of the equations for a binary fluid using a Hamiltonian formalism. Although their derivation was given for an isothermal, inviscid, incompressible fluid, the model was extended to the case where the fluid was viscous and the temperature field varied slowly in time. A similar account can be found in Jacqmin (1996), who discussed the model in a 'potential form' as well as making note of its relation to the above 'stress form'. The merits of these two equivalent formulations were discussed in terms of specific applications. Lowengrub \& Truskinovaky (1997) presented a thorough derivation of the diffuse-interface model based on entropy production and paid particular attention to the difference between 
compressible and quasi-incompressible fluids. The quasi-incompressible situation describes the case where the fluid density is independent of the pressure. However, the bulk states may have different densities and the flow in the interfacial region is in general non-solenoidal $(\nabla \cdot \vec{v} \neq 0)$, resulting in an expansion or contraction flow upon phase transformation. These authors argued that within the context of quasi-incompressibility, the appropriate thermodynamic description is in terms of a Gibbs free energy, in which the pressure is an independent variable determined by the transport equations rather than a quantity determined thermodynamically.

\section{Applications}

THERMOCAPILLARY FLOWS Antanovskii (1995) used his nonisothermal binary fluid model to compute one-dimensional thermocapillary flow in a gap. In this situation, twe fluid phases, characterized by their different compositions, were separated by a planar diffuse interface along which a temperature gradient was imposed. Calculations for different values of interfacial layer thickness and viscosity ratios were presented. Jasnow \& Viñals (1996) investigated thermocapillary migration of droplets of one phase in the surrounding phase. They focused on drops with radii on the order of ten times the correlation length. Their calculations show the motion of the droplet through the temperature gradient as a function of time and the dependence of its velocity on this temperature gradient. Also shown is a sequence in which two droplets coalesce.

SPINODAL DECOMPOSITION Gurtin et al (1996) considered spinodal decomposition occurring in an isothermal binary fluid. Their computations, which begin with an initial random distribution of the composition, show the coarsening process explicitly. They note that the main effect of the hydrodynamic interactions on this process is the flow-induced coalescence of droplets. Their calculations specifically show the flow associated with these coalescence phenomena. They compute the domain (structure) size, compare with classical predictions, and find that for long times the growth is faster than the classical scaling law for coarsening by purely diffusive mechanisms. Jasnow \& Viñals (1996) consider a similar 
situation but include the effects of a nonuniform temperature field. Their results, displayed in the time-sequence in Fig. 3, show the spinodal decomposition of a binary fluid in a rectangular cell across which a vertical temperature gradient is imposed (the cell is hottest at the bottom). Here, an initially random composition distribution (top frame) undergoes coarsening and domain growth. At an intermediate time (middle frame) and a later time (bottom frame), their calculations show nonuniform coarsening wherein the smaller (larger) scales occur in the warmer (cooler) regions.

MIXING AND INTERFACIAL STRETCHING Chella \& Viñals (1996) compute the mixing and interfacial stretching of an isothermal, incompressible binary fluid in a shear flow with equal densities and viscosities in the two phases. An initially planar interface is shown to distort under an imposed shear flow. The wrapping up of the two phases is shown for different values of the capillary number (surface energy). The amount of interfacial stretching is computed and compared with an analytical solution for that associated with a passive scalar. An increase in the surface energy corresponds to a decrease in the amount of stretching and in this way opposes the effect of the shear flow. Further calculaticns by Chella and Viñals of flow in a driven cavity are shown in Fig. 4. A number of breakup and coalescence events can be seen in this sequence.

DROPLET BREAKUP Jacqmin (1996) calculated the breakup of an inviscid fluid in the context of a two-dimensional isothermal model. The initially elongated droplet relaxes, oscillates and breaks apart into two separate droplets. In these calculations, the diffusion coefficient ( $D$ in our notation) was made velocity dependent. In addition to plots showing the actual droplet breakup process, the kinetic and surface energy evolutions are also given. In the case where the flow is strongly damped by using a larger value of $D$, the energy of the droplet decreases rapidly and breakup does not occur.

WAVE-BREAKING AND SLOSHING Jacqmin (1996) also applied the diffuse-interface model to a large-deformation sloshing flow in a two-dimensional rectangular domain. Interaction with the solid wall of the container is modeled using a wall potential associated with a $90^{\circ}$ contact angle between the wall and the interface. The two-phase fluid in a uniform 
(vertical) gravitational field was su'ijected to an oscillating horizontal acceleration whose amplitude increased linearly in time. The motion of th: initially planar interface between the two stably stratified phases is computed once the horizontal accelerations begin. It is shown that the shape of interface becomes highly nonlinear and several breakup and coalescence events can be seen to occur. It is noted that the prediction of coalescence events may occur more quickly than is physically realistic since a high level of accuracy is needed to resolve the draining layer between the two coalescing phases.

MOVING CONTACT LINES Jacqmin (1996) investigated the fluid motion near a moving contact line. These steady calculations show the existence of a dynamic contact angle associated with the interface away from the immediate vicinity of the contact line. Also observed is a streamline (similar to that observed in the experiments by Duss $\cap$ V. \& Davis (1974)) issuing from the contact line region into the displaced fluid.

NIJCLEATION Lowengrub \& Truskinovsky (1997) considered nucleation (and annihilation) of an isothermal, spherically symmetric equilibrium droplet. In particular, they used the diffuse-interface model to describe the situation where the droplet size was comparable to the interface thickness. In the incompressible case, where the density was uniform everywhere, they performed an analysis of the spherically symmetric Cahn-Hilliard equation with a free-energy density composed of piecewise parabolas to find new analytic solutions for the compositions. In the compressible case they proceeded numerically and concluded from their results that compressibility has little effect on the interfacial structure of the droplet.

\section{RELATED TOPICS}

\section{Sharp-Interface Limit}

As noted in Section 2, the diffuse-interface models may be applied away from the critical point, where the interfacial thickness approaches that of a 'sharp' boundary. The use of the diffuse-interface models in these regimes may be justified by demonstrating that they approach asymptotically the free-boundary formulation. This is achieved by adopting what 
is commonly known as the 'sharp-interface limit'.

There has been a significant effort in the context of phase-field models of solidification to address the sharp-interface limit and to compare the model to well-known results of the free-boundary problem (e.g. Caginalp 1989, Braun et al 1994).

One o: the key features in the diffuse-interface models described here, which is not present in the phase-field solidification models studied to date, is the (vector) momentum equation which involves the distributed capillary stresses. Consequently, much of the emphasis in terms of the sharp-interface analyses of the diffuse-interface model has been to recover the classical interfacial boundary conditions associated with the stress balance at the interface. Here we describe the efforts in this area and outline a simple reduction of the momentum balance to the interfacial stress jump using a pillbox argument.

Antanovskii (1995) derived from his diffuse-interface model of a binary fluid the special cases of the classical hydrostatic balance for a flat interface in equilibrium and the YoungLaplace equation for a spherical interface in equilibrium. The latter case has also been considered for a single-component model by Blinowskı (1979). Nadiga and Zaleski (1996) also confirmed numerically that their diffuse-interface model accurately recovered the classical results for a tlat interface and for a liquid droplet in equilibrium. Jasnow and Viñals derived from the capillary term in their momentum equation the appropriate sharp-interface tangential and normal forces when the surface tension was a slowly varying function along the interface. More detailed analytical approaches have been addressed by Starovoitov (1994), Anderson \& McFadden (1996) and Lowengrub \& Truskinovsky (1997).

To illustrate these ideas, we apply a pillbox argument to the momentum balance (17b) to show how the classical stress balance at a fluid-fluid interface can be derived from the diffuseinterface model. We define a small parameter $\epsilon$ measuring the thickness of the interface by $K_{E}=\epsilon^{2} \bar{K}$. We then consider the surface $S_{I}$ defined by the contour of density upon which the interfacial region collapses in the limit $\epsilon \rightarrow 0$ and define a pillbox enclosing a portion of this surface at a fixed point in time in such a way that the top of the pillbox is above the surface at a height $r=\delta$ and the bottom of the pillbox is below the surface at a height $r=-\delta$. 
Here, $r$ is a local coordinate normal to the interface. The key limit in the pillbox argument is that $\epsilon \ll \delta \ll L$ where $L$ is an $O(1)$ length scale associated with the outer flow. In this limit, the volume of the pillbox becomes negligible on the outer scales but the variations in the density, which define the interfacial region, occur over a region fully contained within the pillbox. Also in this limit, the unit normal vectors on the top and bottom of the pillbox are $\hat{n}$ and $-\hat{n}$ respectively while the unit normal on the side is given by $\hat{m}$ (note that $\hat{m} \cdot \hat{n}=0$ ).

We integrate equation (17b) to obtain

$$
0=\int_{V_{p}}\left(\frac{\partial(\rho \vec{v})}{\partial t}+\nabla \cdot(\rho \vec{v} \otimes \vec{v})-\nabla \cdot m\right) d V
$$

where we have used $(17 \mathrm{a})$ and the fact that

$$
\rho \frac{D \vec{v}}{D t}=\frac{\partial(\rho \vec{v})}{\partial t}+\nabla \cdot(\rho \vec{v} \otimes \vec{v})
$$

Next, we note that

$$
\int_{V_{p}} \frac{\partial(\rho \vec{v})}{\partial t} d V \rightarrow-\int_{S_{p}}(\rho \vec{v}) \vec{v}_{I} \cdot \hat{n}_{S} d S
$$

where $\vec{v}_{I}$ is the velocity of the surface $S_{I}$ described above and $S_{p}$ denotes the surface of the pillbox. This result follows by translating to a frame moving with the interface so that $\partial(\rho \vec{v}) / \partial t=\partial(\rho \vec{v}) / \partial t^{\prime}-\vec{v}_{I} \cdot \nabla(\rho \vec{v})=\partial(\rho \vec{v}) / \partial t^{\prime}-\nabla \cdot\left(\rho \vec{v}_{I} \otimes \vec{v}\right)+\rho \vec{v} \nabla \cdot \vec{v}_{I}$. The terms $\partial(\rho \vec{v}) / \partial t^{\prime}+\rho \vec{v} \nabla \cdot \vec{v}_{I}$ are bounded and hence do not contribute as the pillbox volume goes to zero. We use this and the divergence theorem to obtain from equation (22)

$$
0=\int_{S_{p}}\left[\rho \vec{v}\left(\vec{v}-\vec{v}_{I}\right) \cdot \hat{n}_{S}-\hat{n}_{S} \cdot m\right] d S .
$$

We further argue that the fluid velocity terms are bounded ( 80 that they do not contribute to the integral over the side surface of the pillbox) and that the nonclassical terms do not contribute to the upper and lower surfaces of the pillbox (since $\epsilon \ll \delta$ ) so that

$$
0=\int_{A}\left[\rho \vec{v}\left(\vec{v}-\vec{v}_{I}\right) \cdot \hat{n}-\hat{n} \cdot(-p I+\tau)\right]_{-}^{+} d A-\int_{\text {oide }} \hat{m} \cdot T d S
$$

where $A$ is the portion of $S_{I}$ within the pillbox. Now, local to the interfacial region we have $\nabla \rho \sim \rho_{r} \hat{n}$ and $\nabla^{2} \rho \sim \rho_{r r}$ to leading order. Then $\hat{m} \cdot \boldsymbol{T} \sim(\hat{m} \cdot \boldsymbol{T} \cdot \hat{m}) \hat{m}$ so that

$$
0=\int_{A}\left[\rho \vec{v}\left(\vec{v}-\vec{v}_{l}\right) \cdot \hat{n}-\hat{n} \cdot(-p I+T)\right]_{-}^{+} d A-\oint_{C} \int_{-\infty}^{\infty}(\hat{m} \cdot T \cdot \hat{m}) \hat{m} d r d l
$$


where $C$ is the contour defined by the intersection of $S_{I}$ and the pillbox surface and $d l$ is the increment of arclength along $C$. We next define the scalar

$$
\gamma=\int_{-\infty}^{\infty}(\hat{m} \cdot \boldsymbol{T} \cdot \hat{m}) d r
$$

which can be shown to be equal to the excess Kramer's potential, that is, the surface energy (Anderson \& McFadden 1996). We then apply the surface divergence theorem (Weatherburn 1925) which allows us to write

$$
\oint_{C} \gamma \hat{m} d l=\int_{A}\left[\nabla s \gamma-\gamma\left(\nabla_{s} \cdot \hat{n}\right) \hat{n}\right] d A
$$

where $\nabla_{S} \gamma$ is the surface gradient of $\gamma$ and $\nabla_{S} \cdot \hat{n}$, the surface divergence of $\hat{n}$, is the interfacial curvature, $\mathcal{K}$. This gives

$$
0=\int_{A}\left\{\left[\rho \vec{v}\left(\vec{v}-\vec{v}_{I}\right) \cdot \hat{n}-\hat{n} \cdot(-p I+\tau)\right]_{-}^{+}-\left[\nabla_{s \gamma}-\gamma \mathcal{K} \hat{n}\right]\right\} d A
$$

Finally, noting that the area of integration is arbitrary yields

$$
\left[\rho \vec{v}\left(\vec{v}-\vec{v}_{1}\right) \cdot \hat{n}-\hat{n} \cdot(-p I+\tau)\right]_{-}^{+}=\nabla_{s} \gamma-\gamma \mathcal{K} \hat{n}_{1}
$$

which is the classical stress balance at a fluid-fluid interface (Delhaye 1974). Note that the first term on the left-hand side represents a jump in the momentum across the interface and is zero when there is no mass flux across the interface.

\section{Other Computational Methods}

The diffuse-interface models share common features with a number of methods developed from a more computational point of view. We outline several of these methods below and highlight some of their applications.

The volume of fluid (VOF) method (Hirt \& Nichols 1981, Hyman 1984, Tsai \& Yue 1996) is a numerical approach to the free-boundary problem in which an auxiliary function $F$, which distinguishes one fluid from another, is introduced in order to identify the shape and evolution of the free boundary. This function atiafiea $D F / D t=0$; that is, it is advected 
with the flow. Each computational cell has associated with it a value of $F$ and those cells which take on a value between two bulk values of $F$ are assurned to contain part of the interface. The normal to the interface in the cell is determined by the direction of the largest local gradient and the position of the interface in the cell is arranged so that $F$ is the fractional volume of fluid in the cell. The VOF method, like the diffuse-interface approach, allows a straightforward description of flows involving complicated boundary shapes and topological changes. In contrast to the diffuse-interface apr,roach, however, free surface boundary conditions in the VOF method must still be applied at the free boundary.

Brackbill et al (1992) developed a 'continuum surface force' model wherein they identified a volume force which represents surface tension spread over a small but finite threedimensional interfacial domain. This volume force was related to a 'color' function which, for example, can represent density for incompressible flows. The defining characteristics of this volume force are that it gives the correct surface force in the limit of a sharp interface and is nonzero only in the interfacial region. This approach allows a single-domain description of the two-fluid system and does not require the direct application of boundary conditions, which are built into the governing equations. A number of numerical results are presented for both static and dynamic situations: (i) a static drop (rod), (ii) a nonequilibrium rod upon which capillary waves move along the surface, (iii) the Rayleigh-Taylor instability and the associated interfacial deformation, $(i v)$ flow induced by wall-adhesion whereby the fluid conforms to an imposed equilibrium contact angle on the wall, and ( $v$ ) jet-induced tank mixing and liquid reorientation in microgravity environments.

Another related approach has been developed by Unverdi and Tryggvason (1992a, 1992b). Their approach is a front-tracking technique which employs a numerically-diffuse description of the interface. They construct an indicator function, based on the known position of the (sharp) interface, which identifies fluid properties such as density and viscosity. This function is then artificially spread out over a small region on the scale of the computational mesh size, allowing the fluid properties to vary smoothly through this interfacial region. The surface force (i.e. surface tension) is also distributed over this interfacial region so that a single- 
domain approach can be used to calculate the flow. This flow then determines how the interface is advected. In Unverdi and Tryggvason (1992a), both two- and three-dimensional multiple bubble motion and interaction are presented. In Unverdi and Tryggvason (1992b), the Rayleigh-Taylor instability in two and three dimensions and also bubble-bubble interaction in three dimensions are computed. Nobari et al (1996) have recently used this approach to compute head-on collision of two viscous liquid droplets with surface tension. Here, rupture is modeled by artificially removing the thin film between the two drops at a prescribed time. They found that if no rupture takes place, the drops always rebound, but that when rupture occurs the drops may later split. This method has been extended by Juric and Tryggvason $(1995,1996)$ to describe flows in the presence of phase change. Here, they applied the model to vapor bubble dynamics and film boiling.

Another highly successful computational scheme that has been applied to interfacial motion is the level set method, see Osher \& Sethian (1988) and Sethian (1996). With this method, the interface is represented as a level set of a smooth auxiliary function which is computationally analogous to the order parameter used in diffuse-interface descriptions. An advantage of the level set method is that the interface remains sharp in this formulation, which eliminates the need for added numerical resolution in the direction normal to the interface. Within the context of fluid mechanics and two-fluid flows, surface tension, for example, is represented in the momentum equations as a distributed force through the use of a smoothed delta-function (Sussman et al 1994, Chang et al 1996). The momentum equations are then used to compute the flow over the whole domain and the level set function is advected with the flow. After a normalization procedure, the level set function determines the new position of the interface. Mulder et al (1992) applied the level set approach (without surface tension) to study the Rayeigh-Taylor and Kelvin-Helmholtz instabilities within the context of compressible gas dynamics. Sussman et al (1994) used this approach to model incompressible two-dimensional rising bubbles and falling drops, which show large distortions and topological changes. They also show two impacting droplets as well as a single droplet impacting a surface. Chang et al (1996) also applied a level set approach for incompressible 
fluids to several topologically complex flows. They presented computations of two merging fluid bubbles of equal density and also two merging fluid bubbles of different density. Further, they investigated the Rayleigh-Taylor instability of an initially motionless, vertical column of fluid and show calculations of the subsequent vortex sheet roll-up phenomena.

\section{Miscible Fluids}

The ideas that are involved in the d:ffuse-interface models described in the preceding sections are similar in many ways to those for miscible fluids. In particular, fluid stresses (i.e. Korteweg stresses) that arise due to concentration and density gradients at the interface between two miscible fluids lead to the notion of surface tension between miscible fluids. Joseph (1990) has investigated these ideas both experimentally and theoretically. He performed a number of experiments and highlighted other experimental work in which miscible liquid droplets rising or falling in another liquid exhibit capillary-type effects; that is, their shapes are consistent with the presence of surface tension on the interface. In the theoretical development, the equations governing the motion of the fluid are similar to those presented in the above section on binary fluids. They are the continuity equation, the Navier-Stokes equations modified to account for the gradient stresses, the heat (energy) equation, and a standard diffusion equation, rather than the modified Cahn-Hilliard equation (21), that describes the evolution of the composition. Besides the difference in the equations governing the composition, another key difference between the description given by T.igeph (1990) and that for a binary fluid descrit $a$ in section 3 is that his model described a generalized (or quasi) incompressible fluid. Here it is assumed that the density is a function of composition and temperature but is independent of the pressure. Although the density then is unchanged by pressure variations, the astociated flow may atill be nonsolenoidal $(\nabla \cdot \vec{v} \neq 0)$ due to composition or temperature variations. This notion has been carefully adopted into the diffuse-interface model for a binary fluid by Lowengrub \& Truskinovsky (1997).

The theoretical model described by Joseph (1990) has been analyzed in more detail by Galdi et al (1991). These authors rework the equations and identify a new solenoidal velocity 
which is a linear combination of the original velocity field and the concentration gradient. Associated with this new velocity is a pressure field that is a linear combination of the original pressure and the divergence of the original velocity. They address the linear and energy stability of a quiescent, vertically (unstably) stratified incompressible fluid in which Korteweg stresses arise due to composition gradients. This is the analog of the classical Bénard problem with the exception that the authors do not immediately invoke the Boussinesq approximation. They find that the stability results depend strongly on the value of the coefficient in the Korteweg stress (and in particular on its sign). When its sign is chosen consistent with that in the capillary stress term described in Section 2, and when its effect is strong enough, an unconditionally stable base state is predicted.

An notable idea described in the paper by Joseph (1990) is that of a dynamic (or nonequilibrium) surface tension between two miscible fluids. That is, although for two miscible fluids it is not clear that one can define an equilibrium surface tension, based on the idea of stresses associated with gradients in density or composition, transient or dynamic surface tension between two mixing phases can be studied. Joseph et al (1996) consider this situation in detail. In this paper, they carry out an analysis using the model of Joseph (1990) of transient or dynamic interfacial tension during the smoothing of an initial discontinuity of composition across plane and spherical surfaces separating two miscible liquids. It is found that the dynamic interfacial tension decays in time like $t^{-1 / 2}$ and has contributions from Korteweg stresses and from the expansion velocity (i.e. the nonsolenoidal part) which also involves the rate of change of viscosity with composition. For a plane mixing front, diffusion has a similarity solution and they show that there is no associated pressure jump due to the Korteweg terms (i.e. surface tension does not lead to a jump in pressure when the curvature of the interface is zero). The only pressure difference across the mixing front is due to the hydrostatic pressure difference (i.e. due to gravity) across the layer since its thickness is growing with time. For an initially spherical droplet placed in another (miscible) fluid they identify a pressure jump associated with two effects: the first is due to the Korteweg stress and the second is due to the expansion velocity and is proportional to the rate of change of 
viscosity with composition. The latter term can take on either sign, depending on how the viscosity depends on the composition.

\section{SUMMARY}

We have reviewed the development and application of diffuse-interface models for both singlecomponent and binary fluids. These models have foundations in statistical mechanics, kinetic theory, mechanical theories, and nonequilibrium thermodynamics. They provide an alternative approach to the classical hydrodynamic free-boundary problem and have been used successfully in many applications. They should continue to play an important role, in concert with other theoretical and experimental efforts, in the understanding of hydrodynamic phenomena associated with critical fluids and other flows involving complex interface morphologies and topological changes.

\section{ACKNOWLEDGEMENTS}

The authors would like to acknowledge support by the National Aeronautics and Space Administration Microgravity Science and Applications Program. One of the authors (D.M.A.) acknowledges the support of a National Research Council Postdoctoral Fellowship The authors would like to thank S.R. Coriell and B.T. Murray for helpful discussions and P. Seppecher and J. Viñals for providing the figures. 


\section{Literature Cited}

Abraham FF. 1979. On the thermodynamics, structure and phase stability of the non"niform fluid state. Phys. Rep. 53:93-156.

Affouf M, Caflisch RE. 1991. A numerical study of Riemann problem solutions and stability for a system of viscous conservation laws of mixed type. SIAM J. Appl. Math. $51: 605-34$.

Aifantis EC, Serrin JB. 1983a. The mechanical theory of fluid interfaces and Maxwell's rule. J. Colloid Int. Sci. 96:517-29.

Aifantis EC, Serrin JB. 1983b. Equilibrium solutions in the mechanical theory of fluid microstructures, J. Colloid Int. Sci. 96:530-47.

Anderson DM, McFadden GB. 1996. A diffuse-interface description of fluid systems. NIST Int. Rep. 5887, National Institute of Standards and Technology, Gaithersburg, MD.

Anderson DM, McFadden GB. 1997. A diffuse-interface descriptior of internal waves in a near-critical fluid. to appear in Phys. Fluids.

Antanovskii LK. 1995. A phase-field model of capillarity. Phys. Fluids 7:747-53.

Antanovskii LK. 1996. Microscale theory of surface tension. Phys. Rev. E 54:6285-90.

Batchelor GK. 1967. An Introduction to Fluid Dynamics, Cambridge: Cambridge University Press.

Bearman RJ, Kirkwood JG. 1958. Statistical mechanics of transport processes. XI. Equations of transport in multicomponent systers. J. Chem. Phys. 28:136-145.

Berg RF, Lyell MJ, McFadden GB, Rehm RG. 1996. Internal waves in xenon near the critical point. Phys. Fluids 8:1464-75. 
Blinowski A. 1973a. On the surface behavior of gradient-sensitive liquids. Arch. Mech. 25:259-68.

Blinowski A. 1973b. On the order of magnitude of the gradient-of-density dependent part of an elastic potential in liquids. Arch. Mech. 25:833-49.

Blinowski A. 1974. Droplets and layers in the gradient model of a capillary liquid. Arch. Mech. 26:953-63.

Blinowski A. 1975. Gradient description of capillary phenomena in multicomponent fluids. Arch. Mech. 27:273-92.

Blinowski A. 1979. On two phenomenological models of capillary phenomena. Arch. Mech. 31:423-30.

Bongiorno V, Scriven LE, \& Davis HT. 1976. Molecular theory of fluid interfaces. J. Coll. Int. Sci. 57:462-75.

Brackbill JU, Kothe DB, Zemach C. 1992. A continuum method for modeling surface tension. J. Comp. Phys. 100:335-54.

Braun RJ, McFadden GB, Coriell SR. 1994. Morphological instability in phase-field models of solidification. Phys. Rev. E 49:4336-52.

Caginalp G. 1985. Surface tension and supercooling in solidification theory. In Applications of Field Theory to Statistical Mechanics, ed. L Garrido, 216-226 Berlin: Springer-Verlag.

Caginalp G. 1986. An analysis of a phase field model of a free boundary. Arch. Rat. Mech. Anal. 92:205-45.

Caginalp G. 1989. Stefan and Hele-Shaw type models as asymptotic limits of the phase-field equations. Phys. Rev. A 39:5887-96.

Cahn JW. 1961. On spinodal decomposition. Acta Metall. 9:795-801. 
Cahn JW, Hilliard JE. 1958. Free energy of a nonuniform system. I. Interfacial free energy. J. Chem. Phys, 28:258-67.

Callen HB. 1985. Thermodynamics and an Introduction to Thermostatics, New York: Wiley.

Carslaw HS, Jaeger JC. 1959. Conduction of Heat in Solids, Oxford: Clarendon.

Chang YC, Hou TY, Merriman B, Osher S. 1996. A level set formulation of Eulerian interface capturing methods for incompressible fluid flows. J. Camp. Phys. 124:449-64 (1996).

Chella R, Viñals J. 1996. Mixing of a two-phase fluid by cavity flow. Phys. Rev. E 53:3832-40.

Davis HT, Scriven LE. 1982. Stress and structure in fluid interfaces. Adv. Chem. Phys. 49:357-454.

Davis SH. 1983. Contact-line problems in fluid mechanics. J. Appl. Mech. 50:977-82.

de Sobrino L. 1976. Some thermodynamic and stability properties of a fluid with gradient dependent free energy. Can. J. Phys. 54:105-17.

de Sobrino L. 1985. Note on capillary waves in the gradient theory of interfaces. Can. J. Phys. 63:1132-33.

de Sobrino L, Peternelj J. 1982. Thermal fluctuations in the interface of an inhomogeneous fluid. Can. J. Phys. 60:137-53.

de Sobrino L, Peternelj J. 1985. On capillary waves in ihe gradient theory of interfaces. Can. J. Phys. 63:131-34.

Delhaye JM. 1974. Jump conditions and entropy sources in two-phase systems. Local instant formulation. Int. J. Multiphase Flow 1:395-409. 
Dell'Isola F, Gouin H, Rotoli G. 1996. Nucleation of spherical shell-like interfaces by second gradient theory: numerical simulations. Eur. J. Mech. B/Fluids 15:545-68.

Dell'Isola F, Gouin H, Seppecher P. 1995. Radius and surface tension of microscopic bubbles by second gradient theory. C. R. Acad. Sci. Paris 320:211-16.

Dell'Isola F, Kosiński W. 1993. Deduction of thermodynamic balance laws for bidimensional nonmaterial directed continua modelling interphase layers. Arch. Mech. 45:333-59.

Drazin PG, Reid WH. 1981. Hydrodynamic Stability, Cambridge: Cambridge University Press.

Dunn JE. 1986. Interstitial working and a nonclassical continuum thermodynamics. In New Perspectives in Thermodynamics, ed. J Serrin. Berlin: Springer.

Dunn JE, Serrin JB. 1985. On the thermomechanics of interstitial working. Arch. Rat. Mech. Anal. 88:95-133.

Dussan V. EB. 1979. On the spreading of liquids on solid surfaces: static and dynamic contact lines. Ann. Rev. Fluid Mech. 11:371-400.

Dussan V. EB, Davis SH. 1974. On the motion of a fluid-fluid interface along a solid surface. J. Fluid Mech. 65:71-95.

Felderhof BU. 1970. Dynamics of the diffuse gas-liquid interface near the critical point. Physica 48:541-60.

Fixman M. 1967. Transport coefficients in the gas critical region. J. Chem. Phys. 47:2808-18.

Galdi P, Joseph DD, Preziosi L, Rionero S. 1991. Mathematical problems for miscible, incompressible fluj : with Korteweg stresses. Eur. J. Mech. B/Fluids 10(3):253-67. 
Gibbs JW. 1876. On the equilibrium of heterogeneous substances. Trans. Conn. Acad. 3:108-248; 3:343-524, 1878. Reprinted in The Scientific Papers of J. Willard Gibbs, pp. 55-371. London: Longmans, Green, and Co, 1906.

Goldstein H. 1980. Classical Mechanics, Reading, MA, USA: Addison-Wesley.

Gurtin ME, Polignone D, Viñals J. 1996. Two-phase binary fluids and immiscible fluids described by an order parameter. Mathematical Models and Methods in Applied Sciences, 6:815.

Halperin BI, Hohenberg PC, Siggia ED. 1974. Renormalization-group calculations of divergent transport coefficients at critical points. Phys. Rev. Lett. 32:1289-92.

Helfand E, Fredrickson GH. 1989. Large fluctuations in polymer solutions under shear. Phys. Rev. Lett. 62:2468-71.

Hilliard JE. 1970. Spinodal decomposition. In Phase Transformations, ea. HI Aaronson. Metals Park, Ohio: imerican Society of Metals.

Hirt CW, Nichols BD. 1981. Volume of fluid (VOF) methods for the dynamics of free boundaries. J. Comp. Phys. 39:201-25.

Hohenberg PC, Halperin BI. 1977. Theory of dynamic critical phenomena. Rev. Mod. Phys. 49:435-79.

Huh C, Scriven LE. 1971. Hydrodynamic model of steady movement of a solid/liquid/ fluid contact line. J. Coll. Int. Sci. 35:85-101.

Hyman JM. 1984. Numerical methods for tracking interfaces. Physica D 12:396-407. Irving JH, Kirkwood JG. 1950. The statistical mechanical theory of transport processes. IV. The equations of hydrodynamics. J. Chem. Phys. 18:817-829. 
Jacqmin D. 1996. An energy approach to the continuum suriace tension method AIAA 96-0858. In Proceedings of the 34th Aerospace Sciences Meeting and Exhibit. Reno: American Institute of Aeronautics and Astronautics.

Jasnow D, Viñals J. 1996. Coarse-grained description of thermo-capillary flow. Phys. Fluids 8:660-69.

Joseph DD. 1990. Fluid dynamics of two miscible liquids with diffusion and gradient stresses. Eur. J. Mech., B/Fluids 9:565-96.

Joseph DD, Huang A, Hu H. 1996. Non-solenoidal velocity effects and Korteweg stresses in simple mixtures of incompressible liquids. Physica D 97:104-25.

Juric D, Tryggvason G. 1995. A front-tracking method for liquid-vapor phase change. In Advances in Numerical Modeling of Free Surface and Interfacial Fluid Dynamics, ed. PE Raad, TT Huang, G Tryggvason, FED-Vol. 234:141-48. New York: ASME.

Juric D, Tryggvason G. 1996. Direct numerical simulations of flows with phase change. AIAA 96-0857. In Proceedings of the S/th Aerospace Sciences Meeting and Exhibit. Reno: American Institute of Aeronautics and Astronautics.

Kawasaki K. 1970. Kinetic equations and time correlation functions of critical fluct.uations. Ann. Phys. 61:1-56.

Kittel C, Kroemer H. 1980. Thermal Physics, New York: Freeman.

Kobayashi R. 1993. Modeling and numerical simulations of dendritic crystal growth. Physica D 63:410-23.

Korteweg DJ. 1901. Sur la forme que prennent les équations du mouvements des fluides si l'on tient compte des forces capillaires causées par des variations de densité considérables mais continues et sur la théorie de la capillarité dans l'hypothèse d'une variation continue de la densité. Arch. Néerl. Sci. Exactes Nat. Ser. II 6:1-24. 
Lamb H. 1932. Hydrodynamics, Cambridge: Cambridge University Press.

Landau LD, Lifshitz EM. 1959. Fluid Mechanics, New York: Pergamou Press.

Langer JS. 1986. Models of pattern formation in first-order phase transitions. In Directions in Condensed Matter Physics, ed. G Grinstein, G Mazenko, 165-86. ' niladelphia: World Scientific.

Langer JS, Turski LA. 1973. Hydrodynamic model of the condensation of a vapor near its critical point. Phys. Rev. A 8:3230-43.

Lighthill J. 1978. Waves in Fluids, Cambridge: Cambridge University Press.

Lowengrub J, Truskinovsky L. 1997. Cahn-Hilliard fluids and topological transitions. submitted to Proc. Roy. Soc. London A (1997).

Maxwell JC. 1876. Capillary Action. In Encyclopaedia Britannica (9th edition). Reprinted in The Scientific Papers of James Clerk Maxwell, vol. 2, pp. 541-591. New York: Dover, 1952.

Mulder W, Osher S, Sethian JA. 1992. Computing interface motion in compressible gas dynamics. J. Comp. Phys. 100:209-28.

Nadiga BT, Zaleski S. 1996. Investigations of a two-phase fluid model. European J. of Mech. B: Fluids. In press.

Nobari MR, Jan YJ, Tryggvason G. 1996. Head-on collision of drops-a numerical investigation. Phys. Flusids 8:29-42.

Oruki A. 1989. Elastic effects in the phase transition of polymer solutions under shear flow. Phys. Rev. Lett. 62:2472-75.

Onuki A, Kawasaki K. 1979. Nonequilibrium steady state of critical fluids under shear flow: a renormalization group approach. Ann. Phys. 121:456-528. 
Onuki A, Yamazaki K, Kawasaki K. 1981. Light scattering by critical fluids under shear flow. Ann. Phys. 131:217-42.

Osher S, Sethian JA. 1988. Fronts propagating with curvature-dependent speed: algorithms based on Hamilton-Jacobi formulations. J. Comp. Phys. 79:12-49.

Poisson SD. 1831. Nouvelle Théorie de l'action capillaire, Paris: Bachelier.

Lord Rayleigh. 1892. On the theory of surface forces.-II. Compressible fluids. Phil. Mag. 33:209-220.

Rowlinson JS. 1979. Translation of J.D. van der Waals' the thermodynamic theory of capillarity under the hypothesis of a continuous variation of density. J. Stat. Phys. 20:197-244.

Rowlinson JS, Widom B. 1989. Molecular Theory of Capillarity, Oxford: Clarendon.

Seppecher P. 1996. Moving contact lines in the Cahn-Hilliard theory. Int. J. Engng. Sci. 34(9):977-92.

Sethian JA. 1996. Level Set Methods, Cambridge: Cambridge University Press.

Siggia ED, Halperin BI, Hohenberg PC. 1976. Renormalization-group treatment of the critical dynamics of the binary-fluid and gas-liquid transitions. Phys. Rev. $B$ 13:2110-23.

Stanley HE. 1971. Introduction to Phase Transitions and Critical Phenomena, Oxford: Oxford University Press.

Starovoitov VN. 1994. Model of the motion of a two-component liquid with allowance of capillary forces. J. Appl. Mech. Techn. Phys. 35:891-97.

Sussman M, Smerka P, Osher S. 1994. A level set approach for computing solutions to incompressible two-phase flow. J. Comp. Phys. 114:146-59. 
Truskinovsky, L. 1993. Kinks versus shocks. In Shock Induced Transitions and Phase Structures in General Media, IMA Series in Math. and its Appl. ed. JE Dunn, R Fosdick, M Slemrod, 52:185-229. New York: Springer-Verlag.

Tsai W, Yue DKP. 1996. Computation of nonlinear free-surface flows. Ann. Rev. Fluid Mech. 28:249-78.

Turski LA, Langer JS. 1980. Dynamics of a diffuse liquid-vapor interface. Phys. Rev. A 22:2189-95.

Unverdi SO, Tryggvason G. 1992a. A front-tracking method for viscous, incompressible, multi-fluid flows. J. Comp. Phys. 100:25-37.

Unverdi SO, Tryggvason G. 1992b. Computations of multi-fluid flows. Physica $D$ 60:70-83.

van der Waals JD. 1893. Verhandel. Konink. Akad. Weten. Amsterdam (Sect. 1), vol. 1, No. 8 (Dutch); Transl. Ustwald, 1894, Thermodynamische theorie der kapillarität unter voraussetzung stetiger dichteänderung. Z. Phys. Chem. 13:657-725 (Gerniaii); Transl. Anonymous, 1895, Arch. Néerl. 28:121-201 (French). Transl. JS Rowlinson, 1979, Translation of J.D. van der Waals' "The thermodynamic theory of capillarity under the hypothesis of a continuous density variation". J. Stat. Phys. 20:197-244 (From Dutch, German, French)

Wang SL, Sekerka RF, Wheeler AA, Murray BT, Coriell SR, et al. 1993. Thermodynamically-consistent phase-field models for solidification. Physica D 69:189-200.

Warren JA, Boettinger WJ. 1995. Prediction of dendritic growth and microsegregation patterns in a binary alloy using the phase-field method. Acto. metall. mater. 43:689703.

Warren JA, Murray BT. 1996. Ostwald ripening and coalescence of a binary alloy in two dimensions using a phase-field model. Modell. Simul. Mater. Sci. Eng. 4:215-29. 
Weatherburn CE. 1925. Differential invariants in geometry of surfaces, with applications to mathematical physics. Quarterly J. Mathematics 50:230-69.

Wheeler AA, McFadden GB, Boettinger WJ. 1996. Phase-field model for solidification of a eutectic alloy. Proc. Roy. Soc. London A 452:495-525.

Wheeler AA, Murray BT, Schaefer RJ. 1993. Computation of dendrites using a phasefield model. Physica $D$ 66:243-62.

Yang AJM, Fleming III PD, Gibbs JH. 1976. Molecular theory of surface tension. $J$. Chem. Phys. 64:3732-47. 


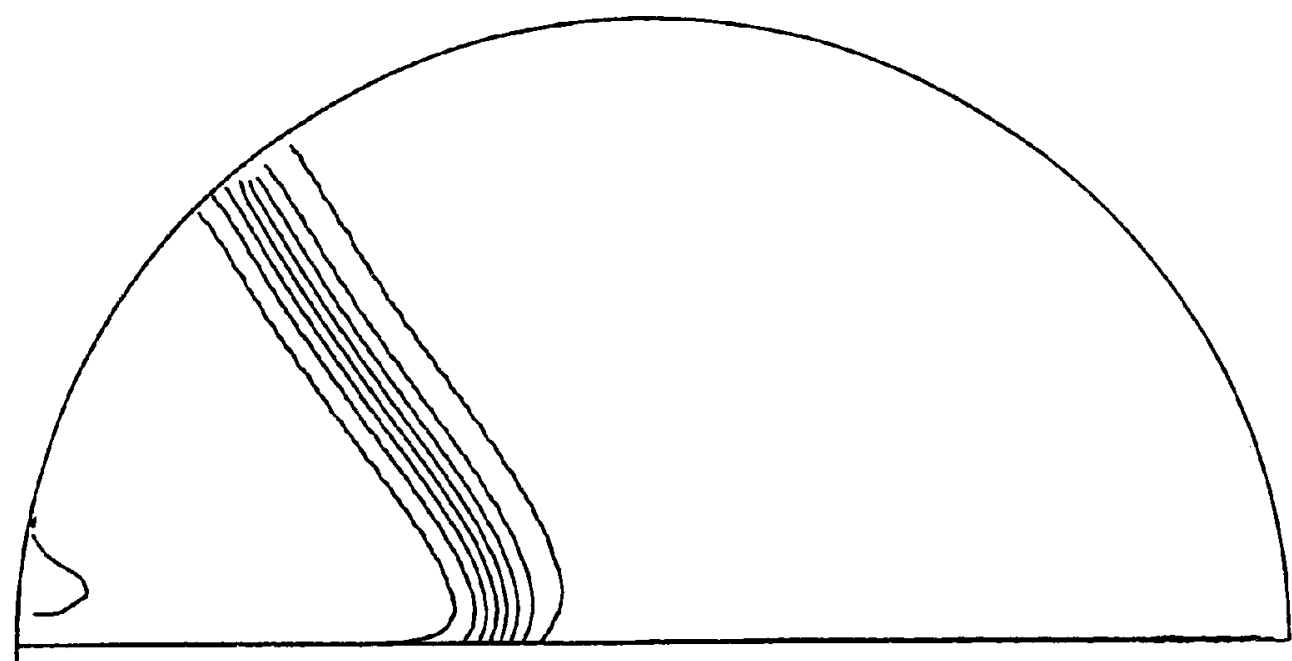

Figure 1: Moving contact line: This figure shows the constant density contours in the inner region. The frame of reference is fixed on the contact line region so that the bottom plate moves from left to right at an imposed velocity. In this case the imposed static contact angle at the wall is approximately $54^{\circ}$ while the dynamic contact angle (away from the immediate vicinity of the contact line) is approximately $125^{\circ}$. The parameter values consistent with the notation given in Seppecher (1996) are $R=20, u_{m}=2, K=10, g=-0.3$, and $C a=40 \times 10^{-3}$. (This figure was provided by $P$. Seppecher). 


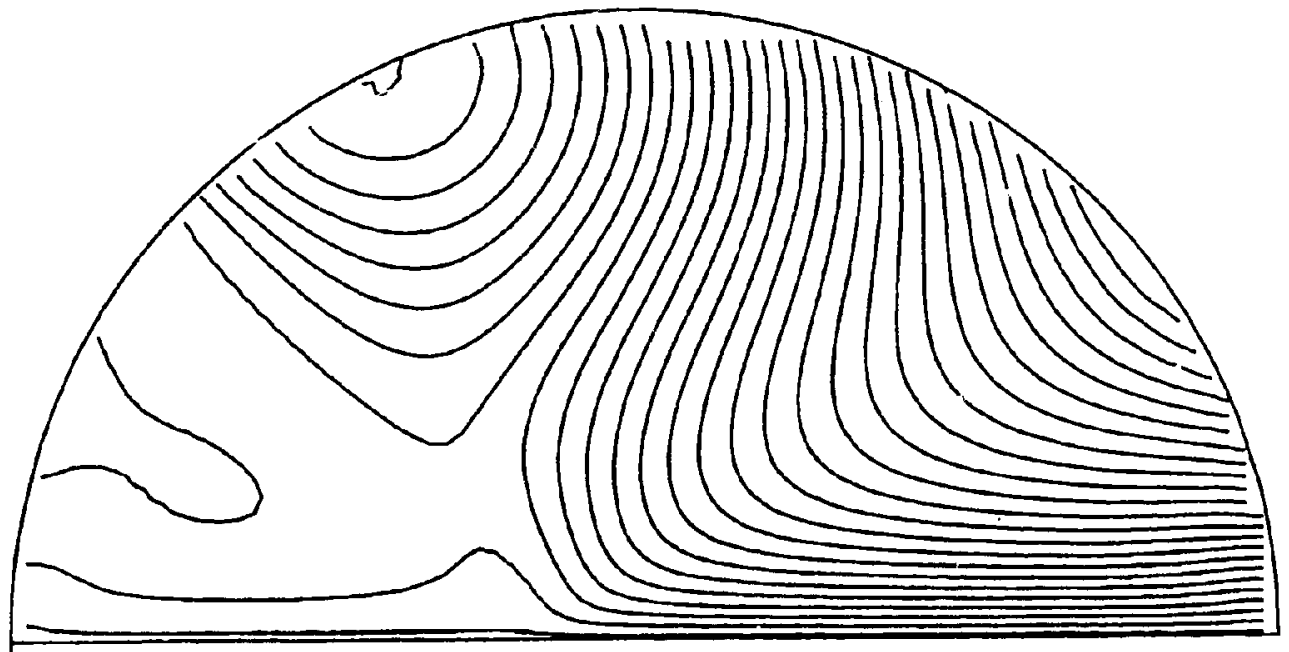

Figure 2: Moving contact line: This figure shows the streamlines in the inner region associated with the density contours shown in Fig. 1. The frame of reference is fixed on the contact line region so that the bottom plate moves from left to right at an imposed velocity. (This figure was provided by P. Seppecher). 


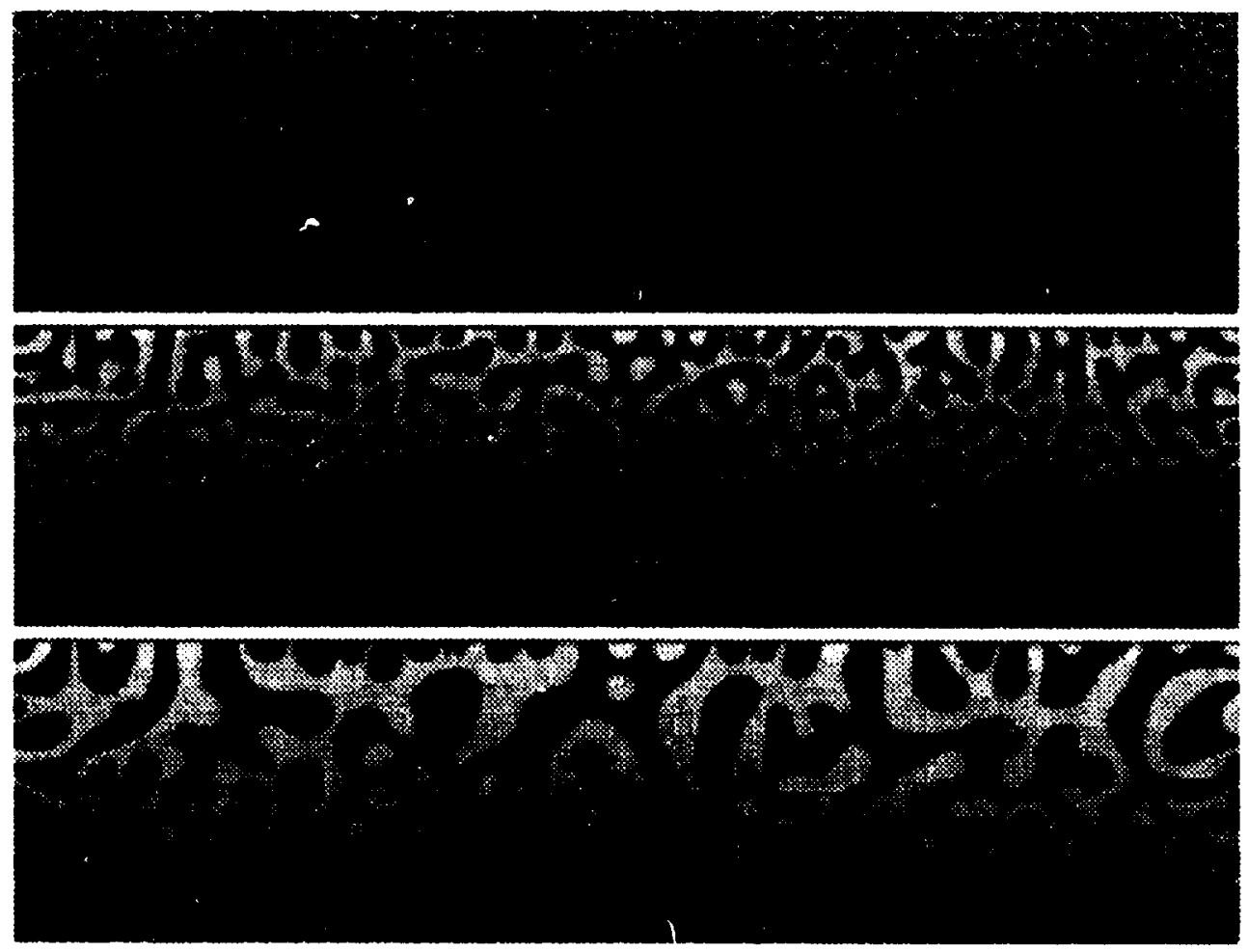

Figure 3: Spinodal decomposition: This time sequence (top to bottom) shows spinodal decomposition of a binary fluid in a rectangular cell across which is imposed a vertical temperature gradient (the cell is hottest at the bottom). The composition is indicated in greyscale. These computations show the system evolving from an initially random composition distribution in the top frame, to one in the bottom trame where the domain structure size varies with the temperature. (This figure was provided by D. Jasnow and J. Viñals). 

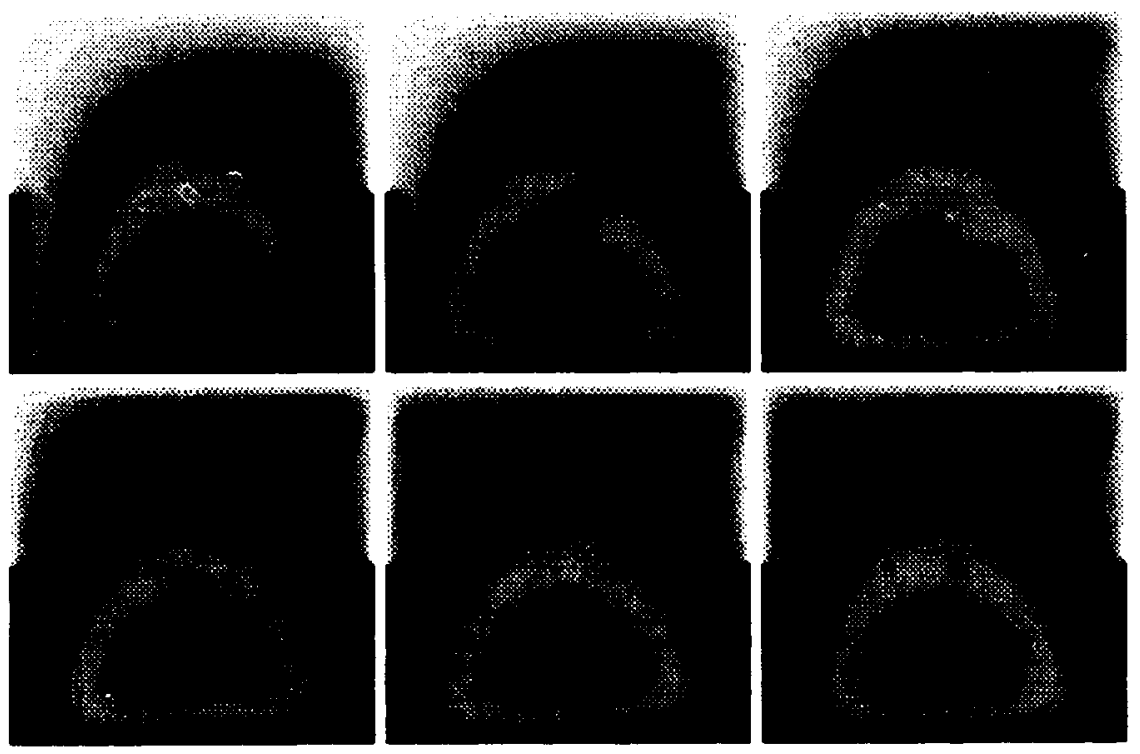

Figure 4: Flow in a driven cavity: This time sequence (across the top, left to right and across the bottom, left to right) shows the order parameter (in greyscale) representing the binary fluid composition. The flow is set up by an imposed velocity (left to right) on the bottom surface of the cavity. The densities and viscosities of both phases are equal. (This figure was provided by R. Chella and J. Viñals). 


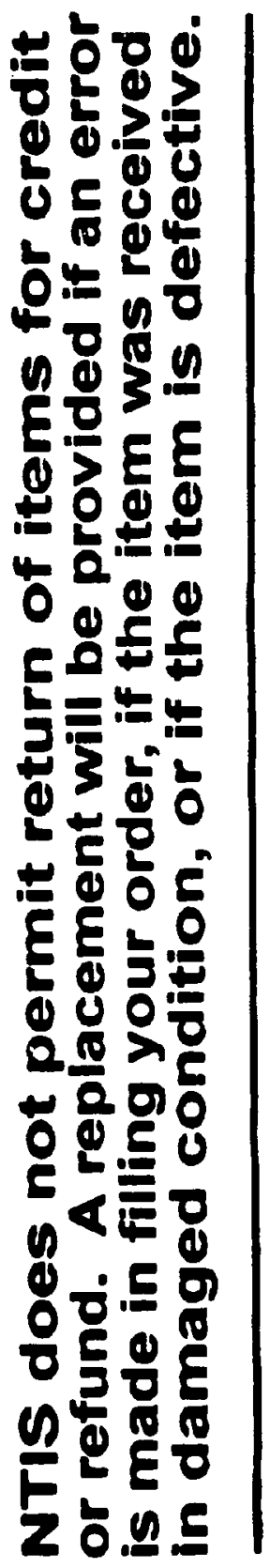

\title{
Reproduced by NTIS
}

\author{
National Technical Information Service \\ Springfield, VA 22161
}

This report was printed specifically for your order

from nearly 3 million tilles available in our collection.

For economy and efficiency, NTIS does not maintain stock of its vast collection of technical reports. Rather, most documents are printed for each order. Documents that are not in electronic format are reproduced from master archival copies and are the best possible reproductions available. If you have any questions concerning this document or any order you have placed with NTIS, please call our Customer Service Department at (703) 487-4660.

\begin{abstract}
About NTIS
NTIS collects scientific, technical, engineering, and business related information - then organizes, maintains, and disseminates that information in a variety of formats - from microfiche to online services. The NTIS collection of nearly 3 million titles includes reports describing research conducted or sponsored by federal agencies and their contractors; statistical and business information; U.S. military publications; audiovisual products; computer software and electronic databases developed by federal agencies; training tools; and technical reports prepared by research organizations worldwide. Approximately 100,000 new titles are added and indexed into the NTIS collection annually.
\end{abstract}

For more information about NTIS products and cervices, call NTIS at (703) $487-4650$ and request the free NTIS Catalog of Products and Services, PR-827LPG, or visit the NTIS Web site http://nnw.ntis.gov.

\author{
NTIS \\ Your indispensable resource for government-sponsoned \\ information-U.S. and wortowide
}

